\title{
Candida albicans HWP1 gene expression and host antibody responses in colonization and disease
}

Correspondence

Paula Sundstrom

paula.sundstrom@dartmouth.edu

Received 23 May 2006

Accepted 6 July 2006

\author{
Julian R. Naglik, ${ }^{1}$ Florentia Fostira, ${ }^{1}$ Jasmeet Ruprai, ${ }^{1}$ Janet F. Staab, ${ }^{2}$ \\ Stephen J. Challacombe ${ }^{1}$ and Paula Sundstrom ${ }^{3}$
}

\begin{abstract}
${ }^{1}$ Department of Oral Medicine and Immunology, King's College London Dental Institute at Guy's, King's College and St Thomas' Hospitals, King's College London, London, UK

${ }^{2}$ Clinical Research Division, Fred Hutchinson Cancer Research Center, 1100 Fairview Ave N, D3-100, Seattle, WA 98109, USA

${ }^{3}$ Department of Microbiology and Immunology, Dartmouth Medical School, Hanover, NH 03755, USA
\end{abstract}

\begin{abstract}
In vivo expression of the developmentally regulated Candida albicans hyphal wall protein 1 (HWP1) gene was analysed in human subjects who were culture positive for $C$. albicans and had oral symptoms $(n=40)$ or were asymptomatic $(n=29)$, or had vaginal symptoms $(n=40)$ or were asymptomatic $(n=29)$. HWP1 mRNA was present regardless of symptoms, implicating hyphal and possibly pseudohyphal forms in mucosal carriage as well as disease. As expected, in control subjects without oral symptoms $(n=10)$ and without vaginal symptoms $(n=10)$ who were culture negative in oral and vaginal samples, HWP1 mRNA was not detected. However, exposure to Hwp1 in healthy culture-negative controls, as well as in oral candidiasis and asymptomatic mucosal infections, was shown by the existence of local salivary and systemic adaptive antibody responses to Hwp1. The results are consistent with a role for Hwp1 in gastrointestinal colonization as well as in mucosal symptomatic and asymptomatic infections. Overall, Hwp1 and hyphal growth forms appear to be important factors in benign and invasive interactions of C. albicans with human hosts.
\end{abstract}

\section{INTRODUCTION}

Candida albicans is a member of the normal flora of the gastrointestinal tract that frequently causes serious oral and vaginal mucosal invasion and systemic disease in hosts with impaired immune defences. For pathogens that persist in the host permanently or for extended periods, understanding the mechanisms that lead to progression from commensalism to virulence is an emerging area of medical research. It has recently become recognized that for pathogens whose ecological niche is the host, molecular factors believed to be important for virulence may also be considered adaptive factors that play an essential role in allowing the pathogen to persist in the host (Falkow, 2006). In these organisms, mechanisms of persistence and virulence may overlap via common determinants that function in both states. For C. albicans, the factors contributing to low-level asymptomatic persistence in the gastrointestinal tract, i.e. colonization, among inhibitory microbial flora, to frequent occurrence of asymptomatic oral and vaginal carriage, and to virulence in immunocompetent and immunodeficient hosts,

Abbreviations: EC, erythematous candidiasis; PC, pseudomembranous candidiasis. are poorly understood. In previous work, we have found that expression of specific secreted aspartyl proteinase (SAP) genes is correlated with active disease and anatomical location, whereas expression of other $S A P$ genes reflects the presence of the organism but not disease (Naglik et al., 2003).

Hyphal wall protein (Hwp1), a transglutaminase substrate which functions as an adhesin, is important for the pathogenesis of candidiasis (Staab et al., 1996, 1999; Sundstrom et al., 2002). HWP1 mRNA and protein are abundant in hyphae in vitro, and greatly reduced or undetectable in yeast forms (Staab et al., 1996, 1999; Sharkey et al., 1999; Nantel et al., 2002). Hypha-specific expression of Hwp1 extends to fungal growth in the host, as shown by the presence and absence of Hwp 1 on hyphae and yeast, respectively, in candidiasis of the murine stomach (Sundstrom et al., 2002). To gain insight into the relationship between HWP1 gene expression and commensalism or symptomatic tissue invasion, we analysed the same carrier and candidiasis specimens that had been used previously to determine SAP and PLB gene expression in samples of whole unstimulated saliva and vaginal swabs (Naglik et al., 2003), for HWP1 gene expression by RT-PCR. Host antibody responses to Hwp1 were also measured. The results 
supported a potentially important role for hyphal forms in asymptomatic infections with $C$. albicans.

\section{METHODS}

Clinical samples. The clinical signs of oral and vaginal candidiasis have previously been described in detail (Naglik et al., 2003). Oral candidiasis included pseudomembranous candidiasis (PC) (thrush), erythematous candidiasis (EC), chronic atrophic candidiasis (CAC), and xerostomia with chronic candidiasis (CC). For vaginal candidiasis, the signs included oedema (swelling), erythema, pseudomembranous plaques, and discharge; and symptoms included pruritus (itch), pain, and soreness. Briefly, samples from individuals who were culture positive for C. albicans (for methods, see Naglik et al., 2005) and symptomatic for oral $(n=40)$ or vaginal $(n=40)$ candidiasis, or culture positive and asymptomatic for oral $(n=29)$ and vaginal $(n=29)$ candidiasis were compared to asymptomatic oral and vaginal culturenegative (from 10 oral and 10 vaginal samples) non-carriers for the presence of HWP1 mRNA. Antifungal therapy had not been administered. Colony counts were $>2 \times 10^{3}$ c.f.u. $\mathrm{ml}^{-1}$ or between 2 and $>10^{4}$ c.f.u. per swab in oral and vaginal candidiasis, respectively, whereas oral carriers and vaginal carriers had $<800$ c.f.u. $\mathrm{ml}^{-1}$ and 4-550 c.f.u. per swab, respectively. The collection of clinical samples was conducted according to the rules of the Guy's and St Thomas' Hospital Trust ethical review board. Informed consent was obtained from all patients regarding the nature of the study.

To determine whether secretory and humoral antibody responses existed in the sample groups, it was necessary to obtain fresh saliva samples from additional subjects, who were classified as patients, carriers and controls according to the above criteria, from individuals attending the Oral Medicine clinic at Guy's Hospital. Serum samples originally collected from some of the individuals in the oral RT-PCR study were supplemented with new samples from patients from the Oral Medicine clinic to obtain adequate numbers for comparison among groups. Sample numbers were based on power calculations from our previous studies (Naglik et al., 1999, 2003).

RT-PCR analysis of C. albicans HWP1 mRNA expression. HWP1 gene expression was examined using qualitative and quantitative RT-PCR. A radioactive qualitative RT-PCR was developed to enable detection of the low levels of C. albicans mRNA in the oral or vaginal carrier state, a requirement for studies aimed at comparing the carrier state with candidiasis (Naglik et al., 1999, 2003). Four RTPCR reactions were performed for each RNA sample prepared from each clinical sample. One reaction to detect HWP1 was performed, accompanied by three different control reactions: an ACT1 control to demonstrate the presence or absence of Candida species, a negative (water) control, and a positive control using genomic DNA isolated from C. albicans NCPF 3156 cells. Each RNA sample was analysed in duplicate, and in many cases in triplicate, to verify HWP1 gene expression. Complete congruence in gene expression was required in two separate analyses using the same RNA sample. RT-PCR experiments using the ACT1 and HWP1 primers were performed using the Access RT-PCR system (Promega). Template RNA was added to RT-

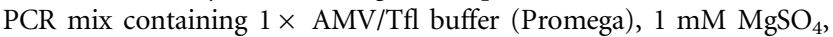
$0.1 \mathrm{mM}$ dNTPs, $0.6 \mu \mathrm{M}$ primers, $3.75 \mathrm{U}$ AMV reverse transcriptase, and $1 \mu \mathrm{Ci}(37 \mathrm{kBq})\left[{ }^{32} \mathrm{P}\right]-\mathrm{dCTP}(\mathrm{ICN})$. Radioactive labelling was used to maximize sensitivity. After reverse transcription $\left(48^{\circ} \mathrm{C}\right.$ for $45 \mathrm{~min}$ ), the sample was denatured at $94^{\circ} \mathrm{C}$ for $3 \mathrm{~min}$, and $2.5 \mathrm{U} \mathrm{Tfl}$ DNA polymerase was added to the reaction (hot start). Cycling times were as follows: denaturation at $94^{\circ} \mathrm{C}$, annealing at $60^{\circ} \mathrm{C}$, and extension at $72{ }^{\circ} \mathrm{C}$, each for $30 \mathrm{~s}$. A final extension at $72^{\circ} \mathrm{C}$ for $10 \mathrm{~min}$ followed cycling. All radiolabelled RT-PCR products were electrophoresed through a $7 \%$ denaturing $7 \mathrm{M}$ urea polyacrylamide gel, exposed to autoradiography film at $-70^{\circ} \mathrm{C}$, and developed. PCR reactions were performed according to the directions of the manufacturer (Promega), incorporating ${ }^{32} \mathrm{P}$ radiolabel as previously described (Naglik et al., 2003). The primers were: HWP1 forward, 5'CCATGTGATGATTACCCACA-3'; and reverse, 5'-GCTGGAACAGAAGATTCAGG-3' (572 bp). Actin (ACT1) [primers: forward, 5'-GATTTTGTCTGAACGTGGTAACAG-3'; and reverse, 5'-GGAGTTGAAAGTGGTTTGGTCAATAC-3' (271 bp)] was utilized as a control gene to detect the presence of $C$. albicans. The authenticity of the amplicons was confirmed by DNA sequencing. Real-time, quantititative RT-PCR was performed with the Qiagen SYBR green kit and the ABI 5700 thermal cycler, following the directions of the manufacturers.

ELISA. Immulon 2 96-well microtitre plates (Thermo Labsystems) were coated with $100 \mu \mathrm{l}$ of $1 \mu \mathrm{g} \mathrm{ml}^{-1}$ purified recombinant Hwp1N13 (rHwp1) consisting of the transglutaminase substrate domain of Hwpl produced as previously described (Staab et al., 2004) antigen in sodium carbonate buffer, $\mathrm{pH} 9 \cdot 6$, for $1 \mathrm{~h}$ at $37^{\circ} \mathrm{C}$. Unused antigen sites were blocked overnight with $200 \mu \mathrm{l}$ PBS/BSA/ Tween 20. For control experiments, the optimum dilution of the rabbit antisera raised against Hwp1 was $1: 8000$. For sample experiments, the optimum dilutions (with minimal background) for each of the stages to carry out the ELISA on individual patient saliva samples were as follows: saliva at $1: 2$, mouse anti-human IgA at $1: 100$, and rabbit anti-mouse-alkaline phosphatase (AP) conjugate at $1: 100$. For patient serum samples, the dilutions were: serum at $1: 15$, mouse anti-human $\operatorname{IgG}$ at $1: 500$, and rabbit anti-mouse-AP at 1:500. All incubations took place for $1 \mathrm{~h}$ at $37^{\circ} \mathrm{C}$, and after each stage the wells were washed three times with PBS. AP enzyme activity was measured by the addition of $100 \mu \mathrm{l} \mathrm{K}$-blue substrate (Neogen). The reaction was stopped by the addition of $100 \mu \mathrm{l} 1 \mathrm{M}$ hydrochloric acid after sufficient blue colour had developed, and was measured in an ELISA reader (Anthos) at $405 \mathrm{~nm}$. Positive controls were rabbit antiserum (R1) generated against rHwp1, and purified serum IgG from a pool of 10 patients with oral candidiasis [using protein G HiTrap columns (Pharmacia) (Naglik et al., 2005)]. For each assay, the negative controls were as follows: no rHwpl coated on the plate; no sample (i.e. saliva or serum); no primary detection antibody. Samples were tested in duplicate and experiments were repeated at least once. IgG and IgA antibody titres to rHwp1 were defined as the inverse of the dilution at which the optical density was twofold greater than background.

\section{RESULTS AND DISCUSSION}

For C. albicans, the relationships between factors that contribute to the three states of existence in the host, (1) low-level asymptomatic persistence, i.e. colonization, in the gastrointestinal tract among inhibitory microbial flora, (2) frequent occurrence of asymptomatic oral and vaginal carriage, and (3) virulence in immunocompetent and immunodeficient hosts, are poorly understood.

In this study, the presence of HWP1 mRNA was found to be correlated with the presence of $C$. albicans in both asymptomatic carriers and in cases of candidiasis at oral and vaginal sites. In the oral cavity, 37/40 individuals with candidiasis and 28/29 carriers were positive for HWP1 mRNA by the radioactive RT-PCR, whereas 59/59 vaginal samples from carriers and candidiasis cases were positive. RT-PCR results for $A C T 1$ and $H W P 1$ were uniformly negative for the culture-negative oral and vaginal control samples.

To quantitate the levels of HWP1 mRNA during oral candidiasis, real-time PCR was performed on samples (five 
per group) from individuals with EC or PC. EC is characterized by a reddening of tissue, but not by the raised white aphthae, consisting of mixtures of fungi and epithelial cells, that are found in PC. An HWP1 message was detected in all samples, with levels that were two- to 20 -fold lower than those of the ACT1 message. Equivalent levels of HWP1 mRNA were found in individuals with PC or EC [PC, median transcript level of 106 (interquartile ranges 106-188); EC, median 128 (interquartile ranges 128-138), relative to an arbitrary transcript level of 1000 for ACT1]. Quantification of HWP1 mRNA from carriers by real-time PCR was attempted, but was inconsistent and nonreproducible, probably because the amount of message was below the necessary threshold for reliable detection by this method (results not shown).

The RT-PCR results showed that carriers and candidiasis cases were equivalent in terms of the expression of HWP1. Furthermore, the five oral specimens in which HWP1 mRNA was not detected were derived from both groups, indicating that cases were not more likely to have HWP1 mRNA than candidiasis carriers. In this respect, $H W P 1$ gene expression results are similar to frequencies seen for SAP2 and SAP5, which are the $S A P$ genes most commonly expressed during candidiasis and carriage (Naglik et al., 1999, 2003). However, in the case of $H W P 1$, the results suggested an increased level of HWP1 mRNA in candidiasis cases. HWP1 mRNA in candidiasis probably exceeded that in asymptomatic conditions, based on the uniformly positive results of RT-PCR in samples from symptomatic, but not asymptomatic, infections. Thus the presence of $H W P 1$ gene expression appears to parallel the tissue burden, as reflected in the higher levels of c.f.u. in the symptomatic group.

Superimposed upon questions regarding mechanisms of persistence in health are the relative contributions of yeasts, hyphae and pseudohyphal growth forms of C. albicans to disease, to carrier states on mucosal surfaces, and to colonization in the lower gastrointestinal tract. The enhanced adherence and invasive properties of hyphal forms relative to yeast forms (Sundstrom, 2006), and the attenuated virulence of mutants unable to form hyphae (Lo et al., 1997), support the belief that equates hyphal forms with invasiveness and yeast forms with commensalism; however, little experimental support exists for this dogma today. Hyphae, pseudohyphae and yeast are found in the same tissue specimens in candidiasis (Sundstrom, 2006), and hyperfilamentous mutants are also attenuated in virulence (Laprade et al., 2002; Bahn et al., 2003), leading to the view that reversible bud-hypha transitions are required for candidiasis. Demonstration of growth morphology during gastrointestinal tract colonization and the carrier state in health is not possible, because of the small numbers of fungi amongst large numbers of normal bacteria in sites that are not easily amenable to specimen collection.

Because HWP1 mRNA is abundant in hyphae compared to yeast, an implication of the presence of HWP1 mRNA in carriers is that hyphal forms are present in the host in the absence of symptoms. This agrees with our previous report, which concluded that hyphae are probably present in most samples, based on widespread expression of SAP4-6 (Naglik et al., 2003). However, HWP1 mRNA may also arise from pseudohyphal growth forms (Snide \& Sundstrom, 2006), or possibly from yeast forms, although experimental data to support the presence of HWP1 mRNA in yeast forms has not been reported.

The high frequency of HWP1 gene expression in oral and vaginal specimens from carriers strongly implicates Hwp1 and hyphae in the ability of $C$. albicans to establish and maintain its presence on mucosal surfaces of human hosts. The results do not support a predominance of yeast forms with absent hyphal forms in the asymptomatic carrier state or in seropositive individuals who lack C. albicans in oral or vaginal specimens. It is also unlikely that PC and EC differ with respect to the morphological composition of $C$. albicans in the tissue, since the measurement of HWP1 gene expression by real-time PCR in EC versus PC showed equivalent levels in both groups.

Specific host determinants that contribute to persistence and candidiasis are also poorly understood. It is not known whether loss of host immunity alone is enough to permit candidiasis, or if upregulation of specific virulence attributes is required. Known immune responses in healthy adults include humoral antibodies to polymannose surface proteins, and cell-mediated immunity to candidal antigens, as demonstrated by positive delayed-type hypersensitivity skin-test reactions to undefined factors in crude extracts that develop early in life (Shannon et al., 1966; Munoz \& Limbert, 1977; Lehmann \& Reiss, 1980).

Table 1. Salivary $\lg A$ and serum $\lg G$ median antibody titres in the three patient populations

Values shown are median antibody titres, with interquartile ranges (IQRs) in parentheses.

\begin{tabular}{|c|c|c|c|c|}
\hline \multirow[t]{2}{*}{ Antibody } & \multicolumn{3}{|c|}{ Patient population } & \multirow[t]{2}{*}{$P$ value $^{\star}$} \\
\hline & Candidiasis & Carrier & Control & \\
\hline Salivary IgA & $13(4 \cdot 0-16 \cdot 0) \quad(n=19)$ & $13 \cdot 5(6 \cdot 0-32 \cdot 0) \quad(n=16)$ & $8(5 \cdot 0-40 \cdot 0) \quad(n=13)$ & $0 \cdot 672$ \\
\hline Serum IgG & $450(405-1105) \quad(n=13)$ & $560(335-1820)(n=13)$ & $415(405-1520) \quad(n=24)$ & $0 \cdot 891$ \\
\hline
\end{tabular}

${ }^{\star}$ Non-parametric Kruskall-Wallis analysis of variance. 
To determine if healthy adults also mount immune responses to Hwp1, antibody titres in oral-culture-negative subjects were compared to those of oral carriers and candidiasis cases by ELISA of rHwp1 (Staab et al., 2004). In contrast to HWP1 gene expression, immune responses to Hwp1 did not correlate with tissue burden in oral samples. Using the non-parametric Kruskall-Wallis analysis of variance, it was found that there were equivalent titres of serum IgG and salivary IgA anti-Hwp1 antibodies in the oral-culture-negative group as well as in the other groups, and there were equivalent titres among the groups also (Table 1). Hwp1, like polymannose, and unlike other antigens that stimulate host responses associated with candidiasis (Strockbine et al., 1984), is a common target of host responses to C. albicans that are recognized to result from long-term colonization. The presence of Hwp1 immune responses in healthy adults does not rule out a role for Hwp1 in the pathogenesis of candidiasis.

The common occurrence of mucosal candidiasis accompanying HIV infection raises the possibility that HIV infection might have affected the antibody responses to Hwpl seen in this study. Although some of the individuals that contributed samples to the RT-PCR results were HIV infected (Naglik et al., 2003), no differences were found between HIV-positive and -negative groups with respect to the presence of Hwp1 mRNA, as detected by RT-PCR. It was not possible to address the effect of HIV infection on salivary anti-Hwp1 IgA levels, because patients providing saliva samples for IgA determinations were not asked about their HIV status. Moreover, those attending the Oral Medicine clinic at Guy's Hospital were deemed to be at low risk for HIV. Given the equivalent anti-Hwp1 antibody levels found in oral-culture-negative controls compared to those of other groups, it is unlikely that the presence of HIV affected the anti-Hwp1 antibody results in this study.

Like the virulence attributes of bacterial pathogens that establish long-term associations with human hosts (Falkow, 2006), Hwp 1 appears to be important both in establishing and maintaining the commensal state, as shown by the universal presence of adaptive immune responses, and in candidiasis, as shown by the probable increase in gene expression in this study, by animal studies (Sundstrom et al., 2002), and by in vitro adherence assays (Staab et al., 1999). Developmental regulation of Hwp1 implicates hyphal and pseudohyphal forms in both commensalism and candidiasis. C. albicans serves as a model organism to elucidate fungal determinants that play a dual role in the establishment of long-term, permanent associations with human hosts, and in tissue invasion leading to disease.

\section{ACKNOWLEDGEMENTS}

This work was supported by a personal Wellcome Trust Value in People (VIP) award to J. R. N., and National Institutes of Health grants
R21 DE015528-01 (J. R. N.) and DE011375 (P. S.). We also thank Ron Wilson for help with statistical analysis. The authors have no conflicting financial interests.

\section{REFERENCES}

Bahn, Y. S., Staab, J. \& Sundstrom, P. (2003). Increased high-affinity phosphodiesterase PDE2 gene expression in germ tubes counteracts CAP1-dependent synthesis of cyclic AMP, limits hypha production and promotes virulence of Candida albicans. Mol Biol 50, 391-409.

Falkow, S. (2006). Is persistent bacterial infection good for your health? Cell 124, 699-702.

Laprade, L., Boyartchuk, V. L., Dietrich, W. F. \& Winston, F. (2002). Spt3 plays opposite roles in filamentous growth in Saccharomyces cerevisiae and Candida albicans and is required for C. albicans virulence. Genetics 161, 509-519.

Lehmann, P. F. \& Reiss, E. (1980). Comparison by ELISA of serum anti-Candida albicans mannan IgG levels of a normal population and in diseased patients. Mycopathologia 70, 89-93.

Lo, H. J., Kohler, J. R., DiDomenico, B., Loebenberg, D., Cacciapuoti, A. \& Fink, G. R. (1997). Nonfilamentous C. albicans mutants are avirulent. Cell 90, 939-949.

Munoz, A. I. \& Limbert, D. (1977). Skin reactivity to Candida and streptokinase-streptodornase antigens in normal pediatric subjects: influence of age and acute illness. J Pediatr 91, 565-568.

Naglik, J. R., Newport, G., White, T. C., Fernandes-Naglik, L. L., Greenspan, J. S., Greenspan, D., Sweet, S. P., Challacombe, S. J. \& Agabian, N. (1999). In vivo analysis of secreted aspartyl proteinase expression in human oral candidiasis. Infect Immun 67, 2482-2490.

Naglik, J. R., Rodgers, C. A., Shirlaw, P. J., Dobbie, J. L., FernandesNaglik, L. L., Greenspan, D., Agabian, N. \& Challacombe, S. J. (2003). Differential expression of Candida albicans secreted aspartyl proteinase and phospholipase B genes in humans correlates with active oral and vaginal infections. J Infect Dis 188, 469-479.

Naglik, J. R., Scott, J., Rahman, D., Mistry, M. \& Challacombe, S. J. (2005). Serum and saliva antibodies do not inhibit Candida albicans Sap2 proteinase activity using a BSA hydrolysis assay. Med Mycol 43, 73-77.

Nantel, A., Dignard, D., Bachewich, C. \& 12 other authors (2002). Transcription profiling of Candida albicans cells undergoing the yeast-to-hyphal transition. Mol Biol Cell 13, 3452-3465.

Shannon, D. C., Johnson, G., Rosen, F. S. \& Austen, K. F. (1966). Cellular reactivity to Candida albicans antigen. N Engl J Med 275, 690-693.

Sharkey, L. L., McNemar, M. D., Saporito-Irwin, S. M., Sypherd, P. S. \& Fonzi, W. A. (1999). HWP1 functions in the morphological development of Candida albicans downstream of EFG1, TUP1, and RBF1. J Bacteriol 181, 5273-5279.

Snide, J. L. \& Sundstrom, P. (2006). Characterisation of HWP1 promoter activation in pseudohyphal cells in Candida albicans. Presentation at the Eighth ASM Conference on Candida and Candidiasis, Denver, CO. Washington, DC: American Society for Microbiology.

Staab, J. F., Ferrer, C. A. \& Sundstrom, P. (1996). Developmental expression of a tandemly repeated, proline- and glutamine-rich amino acid motif on hyphal surfaces on Candida albicans. J Biol Chem 271, 6298-6305.

Staab, J. F., Bradway, S. D., Fidel, P. L. \& Sundstrom, P. (1999). Adhesive and mammalian transglutaminase substrate properties of Candida albicans Hwp1. Science 283, 1535-1538.

Staab, J. F., Bahn, Y. S., Tai, C. H., Cook, P. F. \& Sundstrom, P. (2004). Expression of transglutaminase substrate activity on Candida 
albicans germ tubes through a coiled, disulfide-bonded N-terminal domain of Hwp1 requires C-terminal glycosylphosphatidylinositol modification. J Biol Chem 279, 40737-40747.

Strockbine, N. A., Largen, M. T., Zweibel, S. M. \& Buckley, H. R. (1984). Identification and molecular weight characterization of antigens from Candida albicans that are recognized by human sera. Infect Immun 43, 715-721.
Sundstrom, P. (2006). Candida albicans hypha formation and virulence. In Molecular Principles of Fungal Pathogenesis, pp. 45-47. Washington, DC: American Society for Microbiology.

Sundstrom, P., Balish, E. \& Allen, C. M. (2002). Essential role of the Candida albicans transglutaminase substrate, hyphal wall protein 1 , in lethal oroesophageal candidiasis in immunodeficient mice. J Infect Dis 185, 521-530. 